\section{P-016 INCIDENCE AND MANAGEMENT OF RE-STENOSIS IN PATIENTS STENTED FOR INTRACRANIAL ATHEROSCLEROTIC DISEASE: A CONSECUTIVE SERIES OF 243 STENTED PATIENTS}

M Alexander* , Z Barnard. Department of Neurosurgery, Cedars-Sinai Neurovascular Center, Los Angeles, CA

10.1136/neurintsurg-2019-SNIS.52

Introduction Angioplasty and stenting for intracranial atherosclerotic disease can re-establish adequate luminal diameter in highly stenotic arteries and improve cerebral blood flow. As in other vascular territories, intracranial stented arteries may develop delayed re-stenosis due to progression of atherosclerotic disease or endothelial hyperplasia. The primary strategies for management of re-stenosis have included aggressive medical therapy, angioplasty only, and repeat angioplasty and stenting.

Methods A total of 243 patients underwent intracranial angioplasty and stenting for symptomatic ICAD from 2000-2019 at two institutions. Patients were treated with balloon expandable coronary stents until 2002, when self-expanding intracranial nitinol stents became available. The series included: 21 balloon expandable coronary stents, 36 Neuroform or Atlas stents, 45 Enterprise stents, and 141 Wingspan stents. Of this group, 221 patients had delayed follow up intracranial vascular imaging at 6 months, 1 year, or longer. In follow up, 25 of the 221 patients $(11.3 \%)$ developed significant re-stenosis, defined as $70 \%$ or greater. Eleven of the patients were symptomatic and 14 had no symptoms. A total of 17 patients underwent endovascular treatment of their re-stenosis, and 8 had medical management only.

Results The mean time interval for re-stenosis was 8.7 months. Of the 17 patients treated with endovascular therapy for restenosis, 4 patients had angioplasty only and 13 patients had angioplasty and stenting. There was one peri-procedural stroke in this group (5.9\%) and no other strokes in the follow up period (mean 2.4 years, range 6 months -7 years). Of the 8 patients who were managed medically, 1 patient had a stroke $(12.5 \%)$ in the follow up period at 10 months (mean follow up 2.1 years, range 3 months -5 years).

Discussion The preliminary data from this study suggests that patients with severe re-stenosis may be at significant risk of stroke, even if the re-stenosis is currently asymptomatic. As with other vascular territories, re-stenosis can be managed with multiple modalities, but repeat angioplasty and stenting in this series appears to be a low risk and viable option for these patients compared with medical therapy alone.

Disclosures M. Alexander: 2; C; Stryker Neurovascular. Z. Barnard: None.

\section{P-017 TIME TO INTERVENTION IMPACTS OUTCOMES IN INTRACRANIAL STENTING: ANALYSIS OF 195 CONSECUTIVE PATIENTS IN THE WEAVE TRIAL AND WEAVE REGISTRY COHORTS}

${ }^{1} \mathrm{M}$ Alexander* ${ }^{*}{ }^{2} \mathrm{~J}$ Chaloupka, ${ }^{3} \mathrm{~A}$ Zauner, ${ }^{4} \mathrm{~B}$ Baxter, ${ }^{5} \mathrm{~W}$ Yu. ${ }^{1}$ Department of Neurosurgery, Cedars-Sinai Neurovascular Center, Los Angeles, CA; ${ }^{2} M$ t. Sinai Hospital, Miami, FL; ${ }^{3}$ Department of Neurosurgery, Cottage Hospital, Santa Barbara, CA; ${ }^{4}$ Erlanger Medical Center, Chattanooga, $T N^{5}{ }^{5}$ University of California - Irvine, Irvine, CA

10.1136/neurintsurg-2019-SNIS.53
Introduction The WEAVE Trial was a post-market surveillance study evaluating the peri-procedural stroke and death rate in 152 consecutive patients who were stented with the Wingspan stent under its current on-label FDA indication for symptomatic intracranial atherosclerotic stenosis (ICAS), eight days or longer after stroke. This trial demonstrated a $2.6 \%$ peri-procedural event rate at 72 hours. The WEAVE Registry was a companion registry for 46 consecutive patients stented in an off-label usage of the Wingspan stent at the same centers as the WEAVE Trial patients. The registry demonstrated a $23.4 \%$ peri-procedural event rate that was significantly higher than the on-label trial event rate ( $p$ less than 0.0001).

Methods The peri-procedural event rate of stroke, symptomatic bleed, or death was recorded for all patients in the Trial and the Registry at 72 hours, and the clinical outcomes were verified by core study Neurologists. We evaluated potential risk factors of the stenting procedure to determine which elements were associated with a higher likelihood of peri-procedural complications, including time to stenting from last stroke, and other factors. A total of 195 patients were included in the analysis. The remaining three patients, in whom the qualifying events were not well documented, were excluded.

Results Six of 24 patients (25.0\%) had events who were stented 0 to 7 days post-stroke, six of 69 patients $(8.7 \%)$ had events who were stented 8 to 14 days post-stroke, and 3 of 102 patients $(2.9 \%)$ had events who were stented 15 days or greater following their last stroke. Comparing the event rate in patients who were stented in the recommended time period of 8 days or longer after their last stroke $(5.3 \%)$ to the event rate in patients who were stented early in the $0-7$ day period $(25.0 \%)$, there was a significant difference in clinical outcomes between the two groups $(p=0.0044)$. Other factors did not reach statistical significance independently.

Conclusions Early stenting of patients with symptomatic ICAS was the strongest predictor of poor outcome in the WEAVE Registry cohort. These results indicate that efforts should be made to temporize patients medically until day 8 or longer post-stroke to reduce the peri-procedural morbidity associated with intracranial stenting.

Disclosures M. Alexander: 2; C; Stryker Neurovascular. J. Chaloupka: 2; C; Stryker Neurovascular. A. Zauner: 2; C; Stryker Neurovascular. B. Baxter: 2; C; Stryker Neurovascular. W. Yu: 2; C; Stryker Neurovascular.

\section{P-018 COST EFFECTIVENESS OF ENDOVASCULAR STROKE THERAPY. ANALYSIS FROM A COLOMBIAN HEALTHCARE PERSPECTIVE}

${ }^{1} \mathrm{~B}$ Pabon*, ${ }^{2} \mathrm{~J}$ Tellez, ${ }^{3} \mathrm{~J}$ Arcos, ${ }^{3} \mathrm{P}$ Guijarro, ${ }^{1} \mathrm{C}$ Diaz. ${ }^{1}$ Neuroendovascular Surgery, Angioteam - Clinica del Norte, Medellin, Colombia; ${ }^{2}$ Angioteam, Medellin, Colombia; ${ }^{3}$ Neuroendovascular Surgery, Angioteam, Medellin, Colombia

10.1136/neurintsurg-2019-SNIS.54

Introduction To assess the cost-effectiveness of stent-retriever mechanical thrombectomy in combination with Intravenous Tissue Plasminogen Activator (IV-tPA) versus IV-tPA alone for the Treatment of Acute Ischaemic Stroke in Colombia.

Materials and methods Clinical data were taken from the SWIFT PRIME clinical trial. A lifetime Markov state transition model defined by the modified Rankin Scale score was developed to estimate costs and health outcomes (life years gained 
and quality adjusted life years). A Colombian National Health System perspective (direct medical costs) was considered. Resource utilization was extracted from National Payment Manuals and databases of open access. Costs are expressed in Colombian Pesos (COP). Deterministic and probabilistic sensitivity analyses were performed.

Results Stent-retriever thrombectomy with Intravenous Tissue Plasminogen Activator (IV-tPA) was associated with better outcomes (4.38 life years gained and 2.96 quality adjusted life years) and savings of COP $\$ 1,824,733$ resulting in a dominant therapy over intravenous tissue plasminogen activator alone. A net monetary benefit of COP $\$ 66,005,604$ was obtained considering a willingness-to-pay threshold of COP $\$ 69,081,720$.

Discussion The results were consistent with a previously published cost-effectiveness analysis and reinforce the likeliness of the selection of stent-retriever mechanical thrombectomy plus intravenous tissue plasminogen activator over intravenous tissue plasminogen activator alone.

Conclusion Stent-retriever thrombectomy in combination with Intravenous Tissue Plasminogen Activator is a dominant alternative over intravenous tissue plasminogen activator alone (more effective and less costly) for the treatment of acute ischaemic stroke patients with large vessel occlusions in Colombia.

Disclosures B. Pabon: 2; C; Medtronic. J. Tellez: 5; C; Medtronic. J. Arcos: 5; C; Medtronic. P. Guijarro: 2; C; Medtronic. C. Diaz: None.

\section{P-019 ASPECTS DECAY CAUSING INELIGIBILITY FOR THROMBECTOMY IN PATIENTS TRANSFERRED TO A COMPREHENSIVE STROKE CENTER COVERING A LARGE GEOGRAPHIC AREA}

${ }^{1} S$ Ahmed*, ${ }^{2} \mathrm{E}$ Noyes, ${ }^{3} \mathrm{~K}$ Whelan, ${ }^{2} \mathrm{~B}$ Graham, ${ }^{2} \mathrm{G}$ Hunter, ${ }^{1} \mathrm{M}$ Kelly, ${ }^{1} \mathrm{~L}$ Peeling. ${ }^{1}$ Neurosurgery, University of Saskatchewan, Saskatoon, SK, Canada; ${ }^{2}$ Neurology, University of Saskatchewan, Saskatoon, SK, Canada; ${ }^{3}$ Saskatchewan Cerebrovascular Center, University of Saskatchewan, Saskatoon, SK, Canada

\subsection{6/neurintsurg-2019-SNIS.55}

Introduction Endovascular therapy has revolutionized acute stroke management associated with large vessel occlusion (LVO). The province of Saskatchewan currently has 8 primary stroke centers (PSC) and 1 comprehensive stroke center (CSC) spread over a large geographical area. Seventy three percent of the population lives outside the Saskatoon metropolitan area, which houses the province's single comprehensive stroke centre. Geography remains a significant barrier to providing endovascular therapy in a timely manner to eligible patients, knowing that the time to recanalization is a vital variable in clinical outcome. We aim to quantify the distance and time to transfer of patients to the CSC, and determine factors associated with clinically significant evolution of infarct during transfer.

Methods We completed a retrospective analysis of all acute stroke patients with LVOs who were transferred from PSCs to the CSC for possible endovascular therapy in Saskatchewan from July 2016 - July 2018. Along with patient, imaging, and outcome specific factors, detailed transfer data including modality, time, and distance were collected.

Results Forty-seven patients were identified as having had a LVO diagnosed at a PSC, with subsequent transfer to the
CSC for endovascular therapy. The average age was 70.6 years. Of these patients, 13 (28\%) underwent EVT, while 34 patients did not: 7 (15\%) had clinical improvement, 12 (25\%) had vessel recanalization on repeat CTA, and 15 (32\%) had ASPECTS decay leading to EVT ineligibility. Distance to the PSC and distance from PSC to CSC were the most significant contributors to ASPECTS decay.

Conclusion Rates of ineligibility due to ASPECTS decay were similar to those found in other regional data, despite significantly greater distances. Since the large geographic area covered by the CSC leads to a significant cost burden associated with transfers, we aim to optimize transfer protocols to better serve the outlying areas.

Disclosures S. Ahmed: None. E. Noyes: None. K. Whelan: None. B. Graham: None. G. Hunter: None. M. Kelly: None. L. Peeling: None.

\section{P-020 THE USE OF HF-OCT IN THE PREDICTION OF ANEURYSM OCCLUSION}

${ }^{1} \mathrm{R}$ King ${ }^{*},{ }^{1} \mathrm{M}$ Marosfoi, ${ }^{2} \mathrm{~J}$ Caroff, ${ }^{1} \mathrm{G}$ Ughi, ${ }^{1} \mathrm{D}$ Groth, ${ }^{1} \mathrm{M}$ Gounis, ${ }^{1} \mathrm{~A}$ Puri. ${ }^{1}$ Radiology, University of Massachusetts, Worcester, MA; ${ }^{2}$ Interventional Neuroradiology, Bicetre Hospital, Le Kremlin-Bicêtre, France

\subsection{6/neurintsurg-2019-SNIS.56}

Introduction The development of High Frequency OCT ${ }^{1}$ (HFOCT) allows for higher resolution intra-arterial imaging, such that vascular remodeling in response to stents and next generation intrasaccular devices (NGID) can be interrogated. We hypothesize that imperfections or gaps in the neck coverage of an aneurysm after treatment with a NGID will be predictive of failed aneurysm occlusion.

Materials and methods Two aneurysms were made in each of six dogs, one sidewall aneurysm on the distal right common carotid artery (CCA), and a bifurcation aneurysm proximally at the apex of an anastomosis between the left and right CCAs. One aneurysm was excluded due to thrombosis at the time of treatment. Each aneurysm was treated with either detachable coils, or NGID, with a 7:4 randomization NGID: COIL. At implant, HF-OCT was obtained after each aneurysm was successfully packed. At 180 days, animals were assessed for final aneurysm occlusion by DSA. Separately, the HF-OCT data sets were automatically segmented such that gaps in the coverage of the aneurysm neck could be identified and used to predict if the aneurysm occlusion at 180 days.

Results The overall rate of occlusion was not seen to be different between the NGID and COIL group $(p=0.45)$ on the Raymond-Roy scale. ${ }^{2}$ All of the NGID constructs were successfully segmented, and the associated larges gap in the neck coverage for each was determined. A gap in the NGID construct at the level of the neck greater than $1 \mathrm{~mm}^{2}$ predicted failed aneurysm occlusion at 180-days ( $\mathrm{p}=0.047)$. This threshold correctly classified all aneurysms.

Conclusions HF-OCT allows not only for the imaging and segmentation of intra-arterial devices in vivo, but showed great potential as a robust diagnostic tool. Preliminary evidence collected in this study showed that HF-OCT can prognosticate aneurysm treatment success at the time of treatment.

\section{REFERENCES}

1. Stroke. 2018:Nov29:STROKEAHA118022315

2. Stroke. 2003;34(6):1398-1403. 